\title{
A robust clustering algorithm for analysis of composition-dependent organic aerosol thermal desorption measurements
}

Ziyue Li et al.

Correspondence to: Ziyue Li (liziyue090808@sjtu.edu.cn)

The copyright of individual parts of the supplement might differ from the CC BY 4.0 License. 
The supplemental material includes six tables and six figures, along with additional experimental details for the experiments discussed here.

\section{FIGAERO-CIMS Instrument Description}

The FIGAERO-CIMS instrument has been described previously in detail (Lee et al., 2014; Lopez-Hilfiker et al., 2014). In brief, the measurement of organic aerosol using FIGAERO-CIMS involves two steps: real-time sampling of the gas-phase with simultaneous isothermal collection of particles onto a filter through a separate inlet, followed by temperature programmed thermal desorption and detection of particle-phase species. Thermal desorption of particles occurs in two-stages: a "ramping" and "soaking" period. During ramping, the temperature of UHP $\mathrm{N}_{2}$ programmatically increases from room temperature to $200{ }^{\circ} \mathrm{C}$, typically at $10{ }^{\circ} \mathrm{C} \mathrm{min}$. The majority of the organic aerosol mass desorbs during the ramping stage. During the soaking period, the UHP $\mathrm{N}_{2}$ is held at $200{ }^{\circ} \mathrm{C}$ for ca. 30-40 mins to facilitate evaporation of the remaining, low-volatility organic mass from the filter.

The desorbed gas-phase compounds are transferred to the high-resolution time-of-flight (HRToF) CIMS for continuous detection and quantification at ca. $1 \mathrm{~Hz}$. lodide $\left(\mathrm{I}^{-}\right)$is used as the reagent ion, which is appropriate for characterization of generally highly oxygenated components comprising most secondary organic aerosol (Isaacman-VanWertz et al., 2017; Lee et al., 2018). In a typical SOA system, hundreds of ions having the general formula $\mathrm{C}_{\mathrm{x}} \mathrm{H}_{\mathrm{y}} \mathrm{O}_{\mathrm{z}} \mathrm{l}^{-}$are usually detected. The resulting signal or mass concentration versus temperature (or equivalently time) curves for each ion constitute a thermogram. The overall bulk thermogram is obtained by summing together the individual thermograms.

All individual thermograms are background corrected by subtracting the observed thermograms from appropriate blank experiments. Blank experiments are periodically conducted by placing an additional Teflon filter upstream of the particle filter. The same collection time and desorption processes are used for blank experiments as for samples. The blanks account for contributions from adsorption of gaseous compounds in the air stream and for desorption of compounds from the inner surfaces of the FIGAERO. 


\section{Additional Experimental Details}

Several example applications of the clustering on FIGAERO-CIMS data are discussed in Section 4. These include experiments on SOA derived from: (1) $\mathrm{OH}+\alpha$-pinene and (2) $\mathrm{OH}+$ $\Delta$-3-carene, both at low- $\mathrm{NO}_{x}$ conditions; (3) $\mathrm{OH}+\alpha$-pinene as a function of [NO]; and (4) $\mathrm{O}_{3}+$ $\alpha$-pinene, but where the SOA is allowed to isothermally evaporate for varying amounts of time prior to thermal desorption. These experiments are briefly described below.

All the experiments were done in a $10.6 \mathrm{~m}^{3}$ Teflon environmental chamber at Pacific Northwest National Laboratory (PNNL) (Liu et al., 2012; Liu et al., 2016). Details of SOA formation and chamber conditions are summarized in Table 1.

Experiments \#1-3 were part of the campaign of SOA Formation from Forest Emissions Experiment (SOAFFEE). SOAFFEE was designed and conducted to study the influence of reaction conditions on the formation, composition and properties of biogenic SOA. We consider only a subset of all the SOAFFEE experiments. For the experiments in this study, the chamber operated in continuous-flow mode. The total flow through the chamber was $48.2 \mathrm{~L} \mathrm{~min}^{-1}$, resulting in a residence time of $\sim 3.7$ hours. Biogenic precursors were delivered into the chamber by flushing pure air through a glass bulb immersed in a temperature-controlled liquid bath held at $1^{\circ} \mathrm{C}$ that contained a small volume of the pure liquid. $\mathrm{OH}$ radicals were produced from the photolysis of $\mathrm{H}_{2} \mathrm{O}_{2}$. An aqueous solution of $\mathrm{H}_{2} \mathrm{O}_{2}$ was introduced into a gently warmed glass bulb by a syringe pump. A controlled flow of pure air is passed through the bulb to deliver the desired concentration of $\mathrm{H}_{2} \mathrm{O}_{2}$ into the chamber.

Seed particles of $\left(\mathrm{NH}_{4}\right)_{2} \mathrm{SO}_{4}$ were used to enhance SOA formation and reduce losses of semivolatile reaction products to the chamber walls. Seed particles from atomization were dried and $50 \mathrm{~nm}$ particles were selected using a differential mobility analyzer (DMA), which were introduced into the chamber. The chamber relative humidity $(\mathrm{RH})$ was $50 \%$. For experiments \#12, no NO was added. For experiment \#3, a varying amount of NO was added to the chamber via a calibrated gas cylinder and a mass flow controller. A suite of online instruments characterized the chamber outflow, including a UV absorption $\mathrm{O}_{3}$ analyzer (Thermo Environmental Instruments model $49 \mathrm{C}$ ), an $\mathrm{NO}-\mathrm{NO}_{2}-\mathrm{NO}_{x}$ analyzer (Thermo Environmental models $42 \mathrm{c}$ and $42 \mathrm{i}$ ), a TSI scanning mobility particle sizer for the number and volume concentrations of aerosols (SMPS Model 3081), 
an lonicon quadrupole proton-transfer-reaction mass spectrometer (PTR-QMS) for concentration of precursors, and an Aerodyne high-resolution time-of-flight mass spectrometer (HR-ToF-AMS) for the submicron particle mass and bulk composition. Additionally, FIGAERO-CIMS was used to monitor the gas- and particle-phase products of VOC oxidation.

Experiment \#4 has been described in detail previously (D'Ambro et al., 2018). The work herein focuses on the experiments performed at PNNL at an evaporation $\mathrm{RH}$ of $80 \%$. For this set of experiments, FIGAERO-CIMS was operated in two modes, normal and wait mode. In the normal mode, desorption is initiated as soon as sufficient mass is collected. In the wait mode, collected particles were allowed to isothermally evaporate for some period of time prior to thermal desorption. For the isothermal evaporation, the UHP $\mathrm{N}_{2}$ humidified to $80 \% \mathrm{RH}$ was continuously passed over the filter at room temperature. Dilution of the air around the filter led to evaporation of SOA. The time of isothermal evaporation ranged from 1 hour to 24 hours, resulting in varying extents of mass loss of SOA from the filter. The chemical compositions of the remaining SOA were then characterized by thermal desorption of the particles. 
Table S1 Chemical characteristics of each cluster identified in the $\alpha$-pinene $+\mathrm{OH}$ SOA system

\begin{tabular}{|c|c|c|c|c|c|c|c|c|c|}
\hline Cluster \# & $\begin{array}{c}\text { Expt. \#1 } \\
\text { ( } \alpha \text {-pinene }+\mathrm{OH}) \\
\text { Molecular Formula }\end{array}$ & $\mathrm{O}: \mathrm{C}$ & $\mathrm{H}: \mathrm{C}$ & MW & Mass \% & \# lons & $T_{\mathrm{m}, 50}$ & $T_{\mathrm{m}, 75}$ & $\Delta T$ \\
\hline 1 & $\mathrm{C}_{9.3} \mathrm{H}_{14.9} \mathrm{O}_{3.3} \mathrm{~N}_{0.0}$ & 0.36 & 1.60 & 179.3 & 2.8 & 3 & 86.6 & 110.7 & 24.1 \\
\hline 2 & $\mathrm{C}_{9.6} \mathrm{H}_{16.6} \mathrm{O}_{5.1} \mathrm{~N}_{0.0}$ & 0.53 & 1.72 & 213.4 & 20.1 & 11 & 93.7 & 120.6 & 26.9 \\
\hline 3 & $\mathrm{C}_{8.5} \mathrm{H}_{13.0} \mathrm{O}_{5.1} \mathrm{~N}_{0.0}$ & 0.60 & 1.54 & 196.6 & 44.3 & 42 & 103.7 & 139.2 & 35.5 \\
\hline 4 & $\mathrm{C}_{8.2} \mathrm{H}_{12.9} \mathrm{O}_{6.7} \mathrm{~N}_{0.0}$ & 0.81 & 1.56 & 218.5 & 8.2 & 13 & 110.6 & 140.8 & 30.2 \\
\hline 5 & $\mathrm{C}_{7.4} \mathrm{H}_{10.8} \mathrm{O}_{4.0} \mathrm{~N}_{0.0}$ & 0.54 & 1.46 & 163.6 & 4.0 & 3 & 116.2 & 153.1 & 36.9 \\
\hline 6 & $\mathrm{C}_{11.1} \mathrm{H}_{17.8} \mathrm{O}_{8.1} \mathrm{~N}_{0.0}$ & 0.73 & 1.60 & 280.6 & 0.3 & 6 & 126.2 & 154.5 & 28.3 \\
\hline 7 & $\mathrm{C}_{15.3 .} \mathrm{H}_{22.6} \mathrm{O}_{5.4} \mathrm{~N}_{0.0}$ & 0.35 & 1.48 & 292.6 & 0.2 & 3 & 129.5 & 162.4 & 32.9 \\
\hline 8 & $\mathrm{C}_{8.5} \mathrm{H}_{10.9} \mathrm{O}_{4.0} \mathrm{~N}_{0.0}$ & 0.47 & 1.29 & 176.9 & 1.8 & 2 & 131.0 & 164.1 & 33.1 \\
\hline 9 & $\mathrm{C}_{7.2} \mathrm{H}_{9.4} \mathrm{O}_{4.5} \mathrm{~N}_{0.0}$ & 0.62 & 1.31 & 167.8 & 9.8 & 17 & 131.3 & 163.7 & 32.4 \\
\hline 10 & $\mathrm{C}_{6.9} \mathrm{H}_{7.9} \mathrm{O}_{3.5} \mathrm{~N}_{0.0}$ & 0.50 & 1.15 & 146.7 & 0.6 & 4 & 145.7 & 172.1 & 26.4 \\
\hline 11 & $\mathrm{C}_{4.1} \mathrm{H}_{4.0} \mathrm{O}_{3.9} \mathrm{~N}_{0.0}$ & 0.93 & 0.97 & 115.6 & 0.3 & 2 & 161.8 & 184.2 & 22.4 \\
\hline Unclustered & & & & & 0 & 0 & & & \\
\hline Filtered & & & & & 7.5 & 188 & & & \\
\hline
\end{tabular}




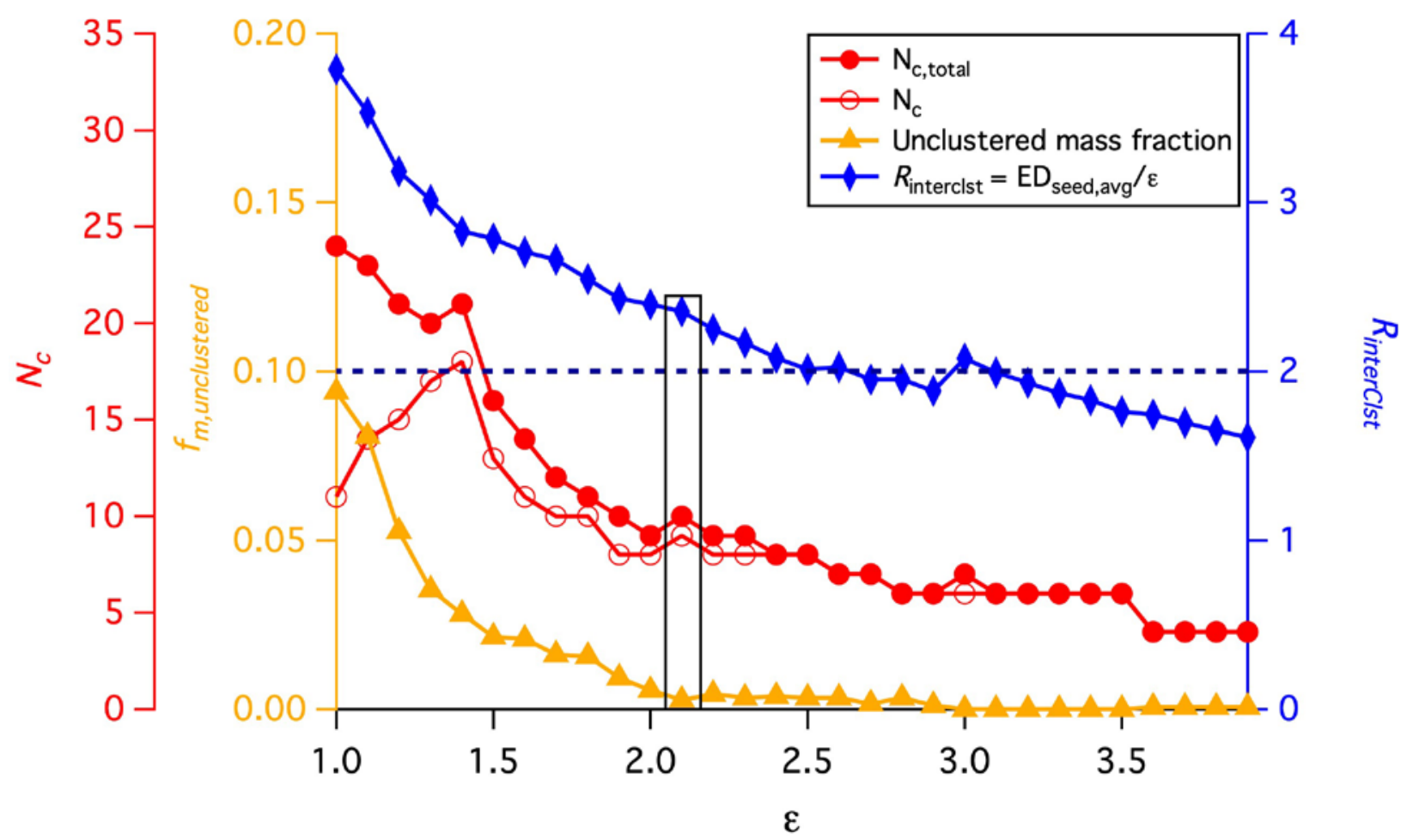

Figure S1. Similar to Figure 4, but guidance for determining the optimal $\varepsilon$ for the $\Delta$-3-carene $+\mathrm{OH}$ SOA. The variation of four parameters, $\mathrm{N}_{c}, \mathrm{~N}_{c, \text { total }}, \mathrm{f}_{\mathrm{m} \text {,unclustered }}$ and $\mathrm{R}_{\text {interclst }}$ are shown as a function of the distance criterion $\varepsilon$. The black horizontal dashed line is guide judgement for $R_{\text {interclst }} \geq 2$. The values highlighted by a rectangle are those corresponding to the optimal $\varepsilon$ used for the following clustering analysis. 
Table S2 Chemical characteristics of each cluster identified in the $\Delta$-3-carene $+\mathrm{OH}$ SOA system

\begin{tabular}{|c|c|c|c|c|c|c|c|c|c|}
\hline Cluster \# & $\begin{array}{c}\text { Expt. \#2 } \\
\text { ( } \Delta \text {-3-carene+ OH) } \\
\text { Molecular Formula }\end{array}$ & $\mathrm{O}: \mathrm{C}$ & $\mathrm{H}: \mathrm{C}$ & MW & Mass \% & \# lons & $T_{\mathrm{m}, 50}$ & $T_{\mathrm{m}, 75}$ & $\Delta T$ \\
\hline 1 & $\mathrm{C}_{9.5} \mathrm{H}_{16.7} \mathrm{O}_{5.7} \mathrm{~N}_{0.0}$ & 0.60 & 1.76 & 221.9 & 16.6 & 3 & 99.4 & 127.0 & 27.6 \\
\hline 2 & $\mathrm{C}_{6.9} \mathrm{H}_{11.3} \mathrm{O}_{4.7} \mathrm{~N}_{0.1}$ & 0.68 & 1.64 & 170.7 & 3.4 & 11 & 102.3 & 138.7 & 36.4 \\
\hline 3 & $\mathrm{C}_{8.3} \mathrm{H}_{12.9} \mathrm{O}_{5.4} \mathrm{~N}_{0.0}$ & 0.65 & 1.55 & 198.9 & 17.5 & 42 & 106.9 & 128.3 & 21.4 \\
\hline 4 & $\mathrm{C}_{8.0} \mathrm{H}_{12.4} \mathrm{O}_{5.3} \mathrm{~N}_{0.0}$ & 0.65 & 1.54 & 193.2 & 28.0 & 13 & 110.1 & 143.9 & 33.8 \\
\hline 5 & $\mathrm{C}_{11.4} \mathrm{H}_{19.7} \mathrm{O}_{8.6} \mathrm{~N}_{0.0}$ & 0.76 & 1.73 & 294.1 & 0.4 & 3 & 120.1 & 147.0 & 26.9 \\
\hline 6 & $\mathrm{C}_{8.2} \mathrm{H}_{11.7} \mathrm{O}_{5.2} \mathrm{~N}_{0.0}$ & 0.63 & 1.42 & 193.3 & 14.8 & 6 & 121.4 & 156.1 & 34.7 \\
\hline 7 & $\mathrm{C}_{6.6} \mathrm{H}_{8.6} \mathrm{O}_{4.6} \mathrm{~N}_{0.0}$ & 0.69 & 1.30 & 161.4 & 6.9 & 3 & 131.3 & 164.6 & 33.3 \\
\hline 8 & $\mathrm{C}_{6.9} \mathrm{H}_{7.9} \mathrm{O}_{3.9} \mathrm{~N}_{0.0}$ & 0.56 & 1.13 & 153.1 & 1.8 & 2 & 141.4 & 170.1 & 28.7 \\
\hline 9 & $\mathrm{C}_{6.0} \mathrm{H}_{7.2} \mathrm{O}_{4.0} \mathrm{~N}_{0.0}$ & 0.68 & 1.21 & 143.2 & 0.3 & 17 & 151.2 & 176.3 & 25.1 \\
\hline 10 & $\mathrm{C}_{4.0} \mathrm{H}_{4.0} \mathrm{O}_{4.0} \mathrm{~N}_{0.0}$ & 1.00 & 1.00 & 116 & 0.6 & 4 & 157.5 & 181.9 & 24.4 \\
\hline Unclustered & & & & & 0.3 & 4 & & & \\
\hline Filtered & & & & & 9.3 & 183 & & & \\
\hline
\end{tabular}




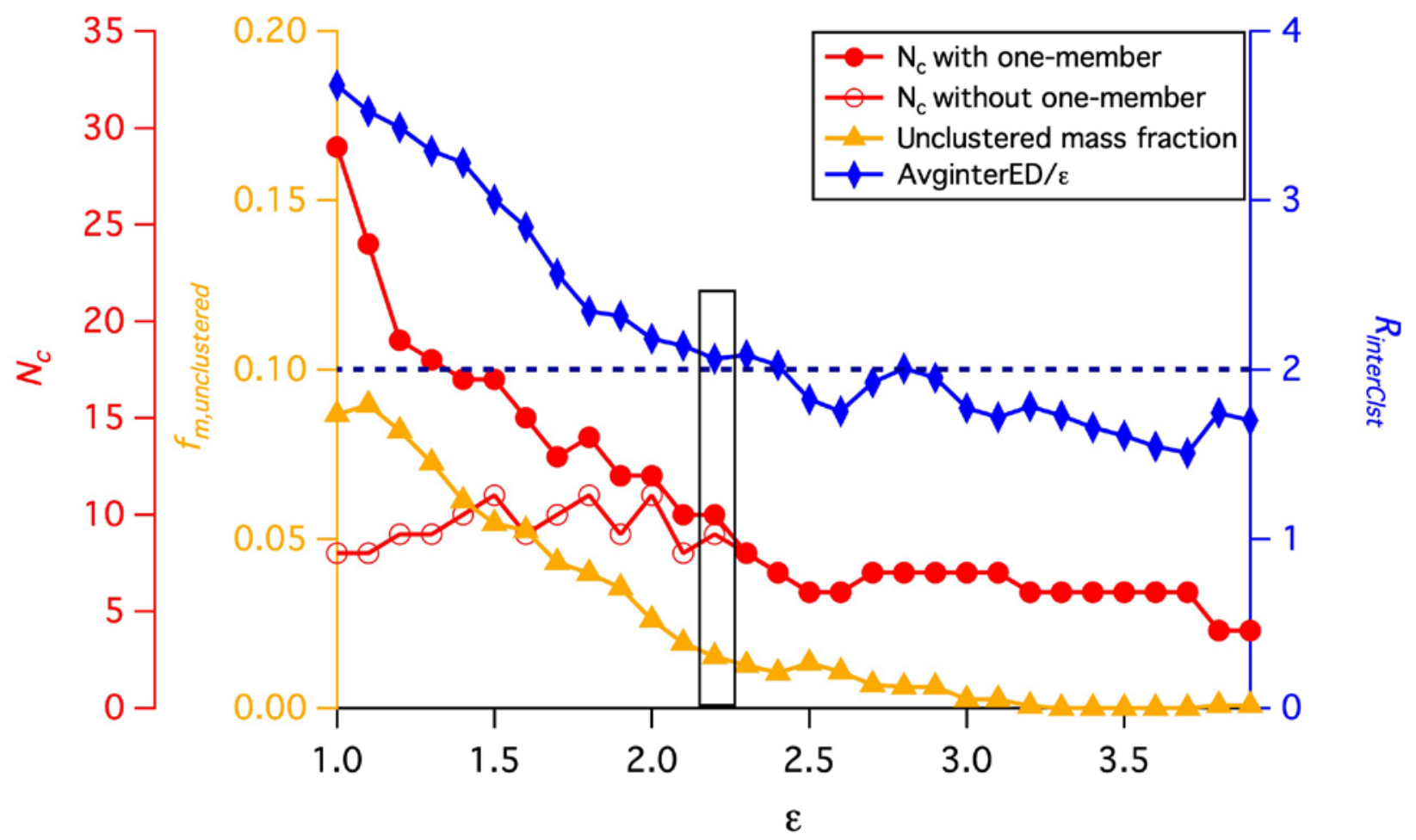

Figure S2. Similar to Figure 4, but guidance for determining the optimal $\varepsilon$ for the $\alpha$-pinene $+\mathrm{OH}$ SOA formed under $5 \mathrm{ppb}$ NO for the single clustering approach. The variation of four parameters, $N_{\mathrm{c}}, N_{\mathrm{c}, \text { total, }}$ $f_{\mathrm{m} \text {,unclustered }}$ and $R_{\text {interclst }}$ are shown as a function of the distance criterion $\varepsilon$. The black horizontal dashed line is guide judgement for $R_{\text {interclst }} \geq 2$. The values highlighted by a rectangle are those corresponding to the optimal $\varepsilon$ used for the following clustering analysis. 

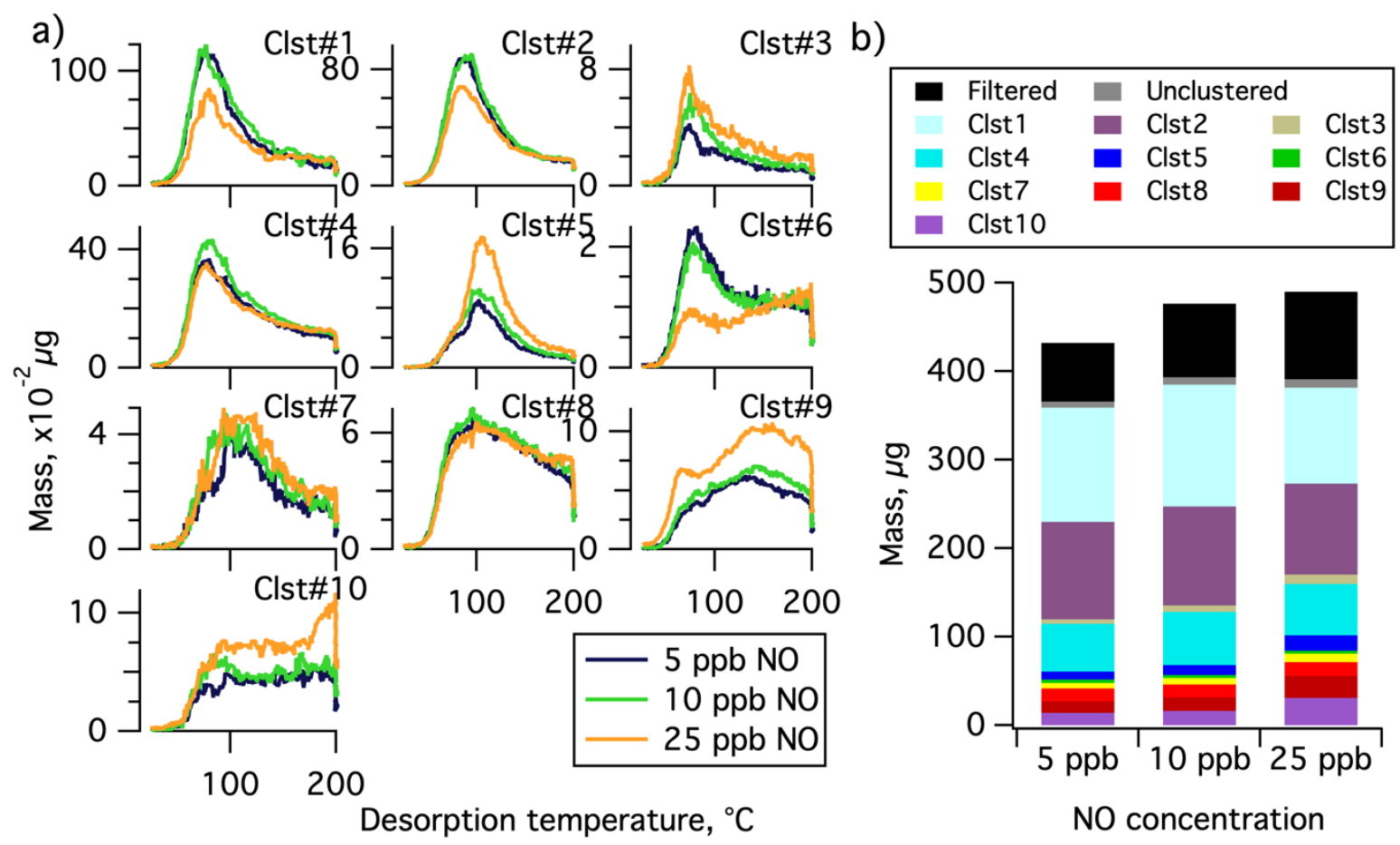

Figure S3 Similar to Figure 9 but presented in the absolute sense. (a) Comparison of the summed thermograms of the 10 clusters of $5 \mathrm{ppb}$ (navy), $10 \mathrm{ppb}$ (green) and $25 \mathrm{ppb}$ (orange) NO experiments. (b) Absolute mass of each cluster for each experiment, including the summed mass of filtered out ions (black) and unclustered ions (gray). 
Table S3. Chemical characteristics of each cluster identified in the $\alpha$-pinene $+\mathrm{OH}+\mathrm{NO}$ SOA system. The single clustering approach is used based on 5 ppb NO experiment.

Table S3-1.

\begin{tabular}{|c|c|c|c|c|c|c|c|c|}
\hline Cluster \# & $\begin{array}{c}\text { Expt. \#3 } \\
\text { ( } \alpha \text {-pinene }+\mathrm{OH}+\mathrm{NO}) \\
\text { Molecular Formula }\end{array}$ & $\mathrm{O}: \mathrm{C}$ & $\mathrm{H}: \mathrm{C}$ & $\mathrm{MW}$ & $\begin{array}{l}{[\mathrm{NO}]} \\
5 \mathrm{ppb}\end{array}$ & $\begin{array}{l}{[\mathrm{NO}]} \\
10 \mathrm{ppb} \\
\text { Mass \% }\end{array}$ & $\begin{array}{l}{[\mathrm{NO}]} \\
25 \mathrm{ppb}\end{array}$ & \# lons \\
\hline 1 & $\mathrm{C}_{8.8} \mathrm{H}_{14.3} \mathrm{O}_{4.7} \mathrm{~N}_{0.0}$ & 0.53 & 1.62 & 195.1 & 29.9 & 28.8 & 22.1 & 27 \\
\hline 2 & $\mathrm{C}_{9.3} \mathrm{H}_{14.8} \mathrm{O}_{6.0} \mathrm{~N}_{0.0}$ & 0.65 & 1.59 & 222.4 & 25.4 & 23.6 & 20.1 & 19 \\
\hline 3 & $\mathrm{C}_{7.5} \mathrm{H}_{10.7} \mathrm{O}_{6.4} \mathrm{~N}_{0.4}$ & 0.85 & 1.42 & 208.7 & 1.2 & 1.4 & 2.1 & 2 \\
\hline 4 & $\mathrm{C}_{8.0} \mathrm{H}_{11.7} \mathrm{O}_{4.9} \mathrm{~N}_{0.1}$ & 0.61 & 1.47 & 187.5 & 12.4 & 12.6 & 11.9 & 10 \\
\hline 5 & $\mathrm{C}_{8.0} \mathrm{H}_{12.0} \mathrm{O}_{6.0} \mathrm{~N}_{0.0}$ & 0.75 & 1.50 & 204.0 & 2.2 & 2.4 & 3.6 & 1 \\
\hline 6 & $\mathrm{C}_{5.5} \mathrm{H}_{7.1} \mathrm{O}_{3.7} \mathrm{~N}_{0.0}$ & 0.67 & 1.28 & 132.3 & 0.9 & 0.7 & 0.7 & 2 \\
\hline 7 & $\mathrm{C}_{8.9} \mathrm{H}_{11.4} \mathrm{O}_{6.3} \mathrm{~N}_{0.0}$ & 0.72 & 1.28 & 219.0 & 1.4 & 1.5 & 1.9 & 4 \\
\hline 8 & $\mathrm{C}_{8.1} \mathrm{H}_{11.1} \mathrm{O}_{4.1} \mathrm{~N}_{0.0}$ & 0.50 & 1.37 & 173.9 & 3.3 & 3.1 & 3.2 & 7 \\
\hline 9 & $\mathrm{C}_{4.6} \mathrm{H}_{5.6} \mathrm{O}_{3.9} \mathrm{~N}_{0.0}$ & 0.83 & 1.20 & 123.2 & 3.0 & 3.1 & 5.1 & 6 \\
\hline 10 & $\mathrm{C}_{8.2} \mathrm{H}_{10.2} \mathrm{O}_{5.0} \mathrm{~N}_{0.0}$ & 0.61 & 1.24 & 188.6 & 3.3 & 3.4 & 6.2 & 2 \\
\hline Unclustered & & & & & 1.5 & 1.7 & 2.0 & 8 \\
\hline Filtered & & & & & 15.5 & 17.5 & 20.2 & 206 \\
\hline
\end{tabular}

Table S3-2.

\begin{tabular}{|c|c|c|c|c|c|c|c|c|c|c|}
\hline $\begin{array}{l}\text { Cluster } \\
\#\end{array}$ & $\begin{array}{c}\text { Expt. \#3 } \\
\text { ( } \alpha \text {-pinene + OH + NO) } \\
\text { Molecular Formula }\end{array}$ & $\begin{array}{l}{[\mathrm{NO}]} \\
5 \mathrm{ppb}\end{array}$ & $\begin{array}{l}{[\mathrm{NO}]} \\
10 \mathrm{ppb} \\
T_{\mathrm{m}, 50}\end{array}$ & $\begin{array}{l}{[\mathrm{NO}]} \\
25 \mathrm{ppb}\end{array}$ & $\begin{array}{l}{[\mathrm{NO}]} \\
5 \mathrm{ppb}\end{array}$ & $\begin{array}{l}{[\mathrm{NO}]} \\
10 \mathrm{ppb} \\
T_{\mathrm{m}, 75}\end{array}$ & $\begin{array}{l}{[\mathrm{NO}]} \\
25 \mathrm{ppb}\end{array}$ & $\begin{array}{l}{[\mathrm{NO}]} \\
5 \mathrm{ppb}\end{array}$ & $\begin{array}{c}{[\mathrm{NO}]} \\
10 \mathrm{ppb} \\
\Delta T\end{array}$ & $\begin{array}{l}{[\mathrm{NO}]} \\
25 \mathrm{ppb}\end{array}$ \\
\hline 1 & $\mathrm{C}_{8.8} \mathrm{H}_{14.3} \mathrm{O}_{4.7} \mathrm{~N}_{0.0}$ & 92.4 & 95.7 & 101.9 & 130.0 & 133.1 & 151.1 & 37.6 & 37.4 & 49.2 \\
\hline 2 & $\mathrm{C}_{9.3} \mathrm{H}_{14.8} \mathrm{O}_{6.0} \mathrm{~N}_{0.0}$ & 100.0 & 100.9 & 103.9 & 136.5 & 136.5 & 145.8 & 36.5 & 35.6 & 41.9 \\
\hline 3 & $\mathrm{C}_{7.5} \mathrm{H}_{10.7} \mathrm{O}_{6.4} \mathrm{~N}_{0.4}$ & 101.8 & 97.1 & 101.8 & 145.9 & 142.2 & 146.1 & 44.1 & 45.1 & 44.3 \\
\hline 4 & $\mathrm{C}_{8.0} \mathrm{H}_{11.7} \mathrm{O}_{4.9} \mathrm{~N}_{0.1}$ & 102.6 & 102.0 & 107.0 & 145.1 & 144.5 & 153.6 & 42.5 & 42.5 & 46.6 \\
\hline 5 & $\mathrm{C}_{8.0} \mathrm{H}_{12.0} \mathrm{O}_{6.0} \mathrm{~N}_{0.0}$ & 108.4 & 109.4 & 114.0 & 138.0 & 137.3 & 139.3 & 29.6 & 27.9 & 25.3 \\
\hline 6 & $\mathrm{C}_{5.5} \mathrm{H}_{7.1} \mathrm{O}_{3.7} \mathrm{~N}_{0.0}$ & 111.1 & 119.9 & 144.3 & 158.2 & 161.3 & 179.0 & 47.1 & 41.4 & 34.7 \\
\hline 7 & $\mathrm{C}_{8.9} \mathrm{H}_{11.4} \mathrm{O}_{6.3} \mathrm{~N}_{0.0}$ & 122.0 & 119.7 & 125.6 & 156.1 & 152.8 & 159.4 & 34.1 & 33.1 & 33.8 \\
\hline 8 & $\mathrm{C}_{8.1} \mathrm{H}_{11.1} \mathrm{O}_{4.1} \mathrm{~N}_{0.0}$ & 125.7 & 125.4 & 129.9 & 160.3 & 160.8 & 166.3 & 34.6 & 35.4 & 36.4 \\
\hline 9 & $\mathrm{C}_{4.6} \mathrm{H}_{5.6} \mathrm{O}_{3.9} \mathrm{~N}_{0.0}$ & 135.9 & 137.4 & 138.9 & 166.4 & 167.1 & 169.3 & 30.5 & 29.7 & 30.4 \\
\hline 10 & $\mathrm{C}_{8.2} \mathrm{H}_{10.2} \mathrm{O}_{5.0} \mathrm{~N}_{0.0}$ & 140.6 & 137.2 & 146.5 & 175.7 & 173.7 & 184.5 & 35.1 & 36.5 & 38.0 \\
\hline $\begin{array}{l}\text { Unclust } \\
\text { Filtered }\end{array}$ & & & & & & & & & & \\
\hline
\end{tabular}



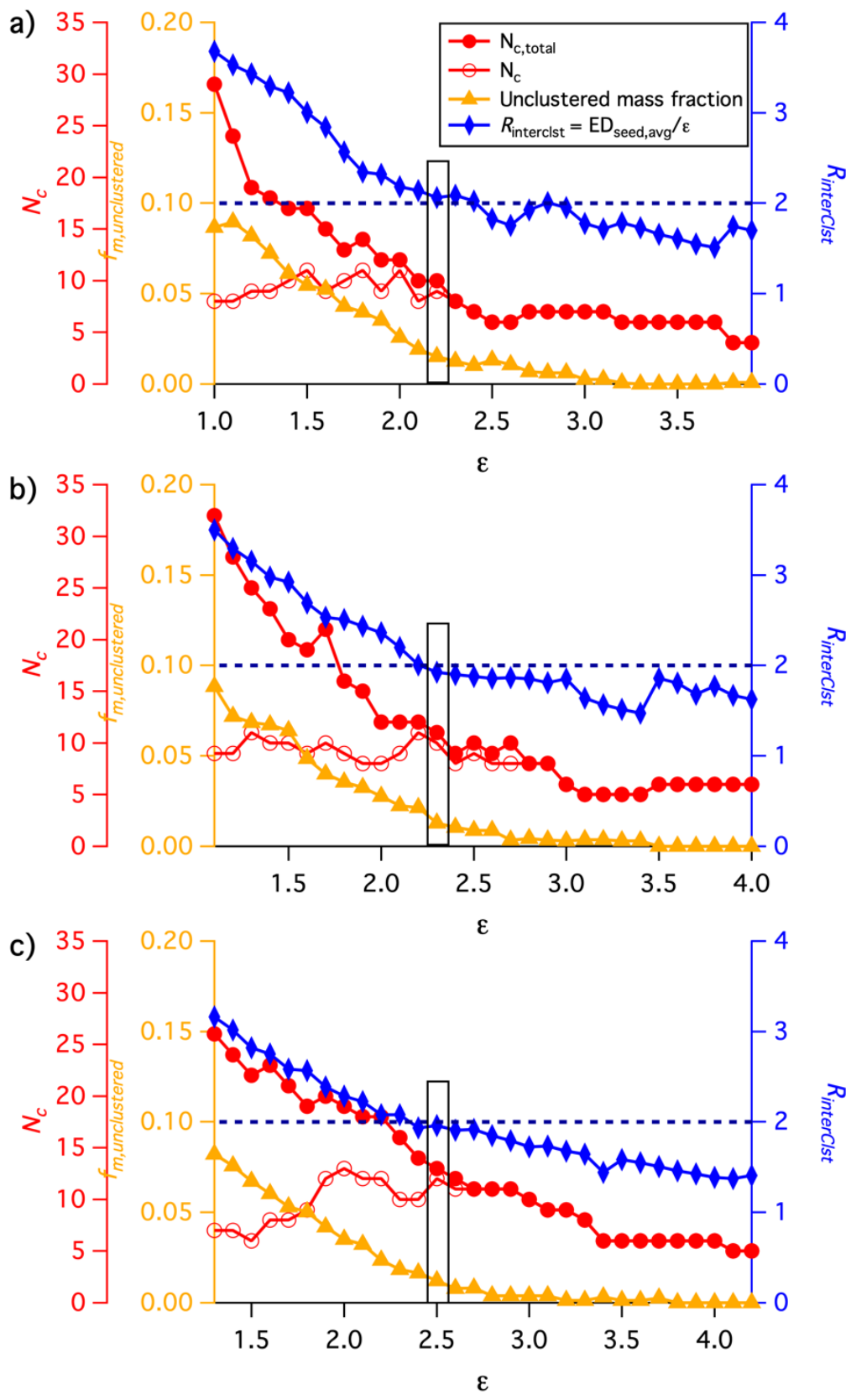

Figure S4. Guidance for determining the optimal $\varepsilon$ for the $\alpha$-pinene $+\mathrm{OH}$ SOA formed under (a) $5 \mathrm{ppb}$, (b) $10 \mathrm{ppb}$ and (c) $25 \mathrm{ppb}$ NO conditions for the multiple clustering approach. The variation of four parameters,

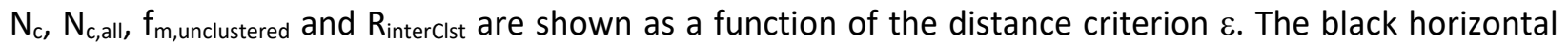
dashed line is guide judgement for $R_{\text {interclst }} \geq 2$. The values highlighted by a rectangle are the values corresponding to the optimal $\varepsilon$ used for the following clustering analysis. 
Table S4 Chemical characteristics of each cluster identified in the $\alpha$-pinene $+\mathrm{OH}+\mathrm{NO}$ SOA system for three different NO conditions (5, 10 and $25 \mathrm{ppb}$ ) from the multiple-clustering approach.

Table S4-1. Results for the $5 \mathrm{ppb}$ NO experiment.

\begin{tabular}{|c|c|c|c|c|c|c|c|c|}
\hline Cluster \# & $\begin{array}{c}\text { Expt. \#3a } \\
\text { ( } \alpha \text {-pinene+ } \mathrm{OH}+5 \text { ppb NO) } \\
\text { Molecular Formula }\end{array}$ & $\mathrm{O}: \mathrm{C}$ & $\mathrm{H}: \mathrm{C}$ & Mass \% & \# lons & $T_{\mathrm{m}, 50}$ & $T_{\mathrm{m}, 75}$ & $\Delta T$ \\
\hline 1 & $\mathrm{C}_{8.8} \mathrm{H}_{14.3} \mathrm{O}_{4.7} \mathrm{~N}_{0.0}$ & 0.53 & 1.62 & 29.9 & 27 & 92.4 & 130.0 & 37.6 \\
\hline 2 & $\mathrm{C}_{9.3} \mathrm{H}_{14.8} \mathrm{O}_{6.0} \mathrm{~N}_{0.0}$ & 0.65 & 1.59 & 25.4 & 19 & 100.0 & 136.5 & 36.5 \\
\hline 3 & $\mathrm{C}_{7.5} \mathrm{H}_{10.7} \mathrm{O}_{6.4} \mathrm{~N}_{0.4}$ & 0.85 & 1.42 & 1.2 & 2 & 101.8 & 145.9 & 44.1 \\
\hline 4 & $\mathrm{C}_{8.0} \mathrm{H}_{11.7} \mathrm{O}_{4.9} \mathrm{~N}_{0.1}$ & 0.61 & 1.47 & 12.4 & 10 & 102.6 & 145.1 & 42.5 \\
\hline 5 & $\mathrm{C}_{8.0} \mathrm{H}_{12.0} \mathrm{O}_{6.0} \mathrm{~N}_{0.0}$ & 0.75 & 1.50 & 2.2 & 1 & 108.4 & 138.0 & 29.6 \\
\hline 6 & $\mathrm{C}_{5.5} \mathrm{H}_{7.1} \mathrm{O}_{3.7} \mathrm{~N}_{0.0}$ & 0.68 & 1.28 & 0.9 & 2 & 111.1 & 158.2 & 47.1 \\
\hline 7 & $\mathrm{C}_{8.9} \mathrm{H}_{11.4} \mathrm{O}_{6.3} \mathrm{~N}_{0.0}$ & 0.72 & 1.28 & 1.4 & 4 & 122.0 & 156.1 & 34.1 \\
\hline 8 & $\mathrm{C}_{8.1} \mathrm{H}_{11.1} \mathrm{O}_{4.1} \mathrm{~N}_{0.0}$ & 0.50 & 1.37 & 3.3 & 7 & 125.7 & 160.3 & 34.6 \\
\hline 9 & $\mathrm{C}_{4.6} \mathrm{H}_{5.6} \mathrm{O}_{3.9} \mathrm{~N}_{0.0}$ & 0.83 & 1.20 & 3.0 & 6 & 135.9 & 166.4 & 30.5 \\
\hline 10 & $\mathrm{C}_{8.2} \mathrm{H}_{10.2} \mathrm{O}_{5.0} \mathrm{~N}_{0.0}$ & 0.61 & 1.24 & 3.3 & 2 & 140.6 & 175.7 & 35.1 \\
\hline Unclustered & & & & 1.5 & 8 & & & \\
\hline Filtered & & & & 15.5 & 208 & & & \\
\hline
\end{tabular}

Table S4-2. Results for the $10 \mathrm{ppb}$ NO experiment.

\begin{tabular}{|c|c|c|c|c|c|c|c|c|}
\hline Cluster \# & $\begin{array}{c}\text { Expt. \#3b } \\
\text { ( } \alpha \text {-pinene+ } \mathrm{OH}+10 \mathrm{ppb} \mathrm{NO}) \\
\text { Molecular Formula }\end{array}$ & $\mathrm{O}: \mathrm{C}$ & $\mathrm{H}: \mathrm{C}$ & Mass $\%$ & \# lons & $T_{\mathrm{m}, 50}$ & $T_{\mathrm{m}, 75}$ & $\Delta T$ \\
\hline 1 & $\mathrm{C}_{8.5} \mathrm{H}_{13.6} \mathrm{O}_{4.9} \mathrm{~N}_{0.1}$ & 0.58 & 1.60 & 27.6 & 28 & 95.1 & 134.1 & 39.0 \\
\hline 2 & $\mathrm{C}_{9.1} \mathrm{H}_{14.3} \mathrm{O}_{6.1} \mathrm{~N}_{0.1}$ & 0.66 & 1.57 & 25.7 & 21 & 101.9 & 136.5 & 34.6 \\
\hline 3 & $\mathrm{C}_{8.5} \mathrm{H}_{12.8} \mathrm{O}_{4.9} \mathrm{~N}_{0.1}$ & 0.58 & 1.50 & 17.3 & 14 & 102.1 & 143.3 & 41.2 \\
\hline 4 & $\mathrm{C}_{5.4} \mathrm{H}_{11.1} \mathrm{O}_{7.6} \mathrm{~N}_{0.2}$ & 1.40 & 2.06 & 2.1 & 4 & 104.1 & 148.4 & 44.3 \\
\hline 5 & $\mathrm{C}_{6.8} \mathrm{H}_{8.4} \mathrm{O}_{4.6} \mathrm{~N}_{0.0}$ & 0.68 & 1.23 & 3.2 & 5 & 110.9 & 159.5 & 48.6 \\
\hline 6 & $\mathrm{C}_{12.5} \mathrm{H}_{18.9} \mathrm{O}_{7.5} \mathrm{~N}_{0.0}$ & 0.60 & 1.52 & 0.2 & 2 & 124.5 & 152.9 & 28.4 \\
\hline 7 & $\mathrm{C}_{9.7} \mathrm{H}_{14.3} \mathrm{O}_{7.3} \mathrm{~N}_{0.0}$ & 0.75 & 1.47 & 0.3 & 2 & 126.5 & 159.3 & 32.8 \\
\hline 8 & $\mathrm{C}_{8.2} \mathrm{H}_{11.0} \mathrm{O}_{4.2} \mathrm{~N}_{0.0}$ & 0.51 & 1.35 & 3.1 & 7 & 126.3 & 161.1 & 34.8 \\
\hline 9 & $\mathrm{C}_{4.0} \mathrm{H}_{4.0} \mathrm{O}_{6.0} \mathrm{~N}_{0.0}$ & 1.50 & 1.00 & 0.5 & 1 & 134.3 & 168.5 & 34.2 \\
\hline 10 & $\mathrm{C}_{7.8} \mathrm{H}_{9.5} \mathrm{O}_{4.8} \mathrm{~N}_{0.0}$ & 0.61 & 1.23 & 4.1 & 2 & 137.8 & 172.4 & 34.6 \\
\hline 11 & $\mathrm{C}_{3.2} \mathrm{H}_{3.8} \mathrm{O}_{3.9} \mathrm{~N}_{0.0}$ & 1.25 & 1.22 & 1.9 & 4 & 138.6 & 166.3 & 27.7 \\
\hline Unclustered & & & & 1.3 & 10 & & & \\
\hline Filtered & & & & 12.6 & 195 & & & \\
\hline
\end{tabular}


Table S4-3. Results for the $25 \mathrm{ppb}$ NO experiment.

\begin{tabular}{lllllllll}
\hline Cluster \# & $\begin{array}{c}\text { Expt. \#3c } \\
\text { (a-pinene+ OH+25 ppb NO) } \\
\text { Molecular Formula }\end{array}$ & O:C & H:C & Mass \% & \# lons & $T_{\mathrm{m}, 50}$ & $T_{\mathrm{m}, 75}$ & $\Delta T$ \\
\hline 1 & $\mathrm{C}_{10.0} \mathrm{H}_{15.0} \mathrm{O}_{6.0} \mathrm{~N}_{1.0}$ & 0.60 & 1.50 & 1.1 & 1 & 92.3 & 139.8 & 47.5 \\
2 & $\mathrm{C}_{6.0} \mathrm{H}_{8.8} \mathrm{O}_{5.0} \mathrm{~N}_{0.0}$ & 0.82 & 1.46 & 0.8 & 4 & 98.5 & 147.8 & 49.3 \\
3 & $\mathrm{C}_{8.1} \mathrm{H}_{12.4} \mathrm{O}_{5.4} \mathrm{~N}_{0.2}$ & 0.66 & 1.53 & 39.8 & 29 & 103.4 & 150.7 & 47.3 \\
4 & $\mathrm{C}_{9.1} \mathrm{H}_{14.1} \mathrm{O}_{6.3} \mathrm{~N}_{0.1}$ & 0.69 & 1.55 & 13.7 & 18 & 106.5 & 144.4 & 37.9 \\
5 & $\mathrm{C}_{6.1} \mathrm{H}_{9.8} \mathrm{O}_{6.3} \mathrm{~N}_{0.0}$ & 1.03 & 1.60 & 3.7 & 2 & 121.2 & 160.9 & 39.7 \\
6 & $\mathrm{C}_{8.7} \mathrm{H}_{11.3} \mathrm{O}_{6.2} \mathrm{~N}_{0.0}$ & 0.72 & 1.30 & 3.2 & 5 & 122.2 & 155.0 & 32.8 \\
7 & $\mathrm{C}_{7.9} \mathrm{H}_{10.7} \mathrm{O}_{4.8} \mathrm{~N}_{0.1}$ & 0.61 & 1.36 & 2.8 & 8 & 125.1 & 165.1 & 40.0 \\
8 & $\mathrm{C}_{4.0} \mathrm{H}_{5.6} \mathrm{O}_{3.9} \mathrm{~N}_{0.0}$ & 0.97 & 1.37 & 1.7 & 5 & 134.9 & 168.6 & 33.7 \\
9 & $\mathrm{C}_{11.8} \mathrm{H}_{18.5} \mathrm{O}_{7.7} \mathrm{~N}_{0.0}$ & 0.65 & 1.57 & 0.2 & 3 & 136.5 & 166.2 & 29.7 \\
10 & $\mathrm{C}_{2.5} \mathrm{H}_{3.0} \mathrm{O}_{4.0} \mathrm{~N}_{0.0}$ & 1.58 & 1.20 & 2.3 & 3 & 139.2 & 168.7 & 29.5 \\
11 & $\mathrm{C}_{5.2} \mathrm{H}_{5.8} \mathrm{O}_{5.1} \mathrm{~N}_{0.0}$ & 0.97 & 1.11 & 3.8 & 3 & 140.2 & 175.5 & 35.3 \\
12 & $\mathrm{C}_{7.8} \mathrm{H}_{9.7} \mathrm{O}_{4.9} \mathrm{~N}_{0.0}$ & 0.63 & 1.25 & 1.0 & 2 & 147.7 & 185.4 & 37.7 \\
13 & $\mathrm{C}_{4.2} \mathrm{H}_{4.0} \mathrm{O}_{3.8} \mathrm{~N}_{0.0}$ & 0.92 & 0.96 & 1.5 & 2 & 167.5 & 188.9 & 21.4 \\
Unclustered & & & & 0.7 & 7 & & & \\
Filtered & & & & 16.0 & 205 & & & \\
\hline
\end{tabular}




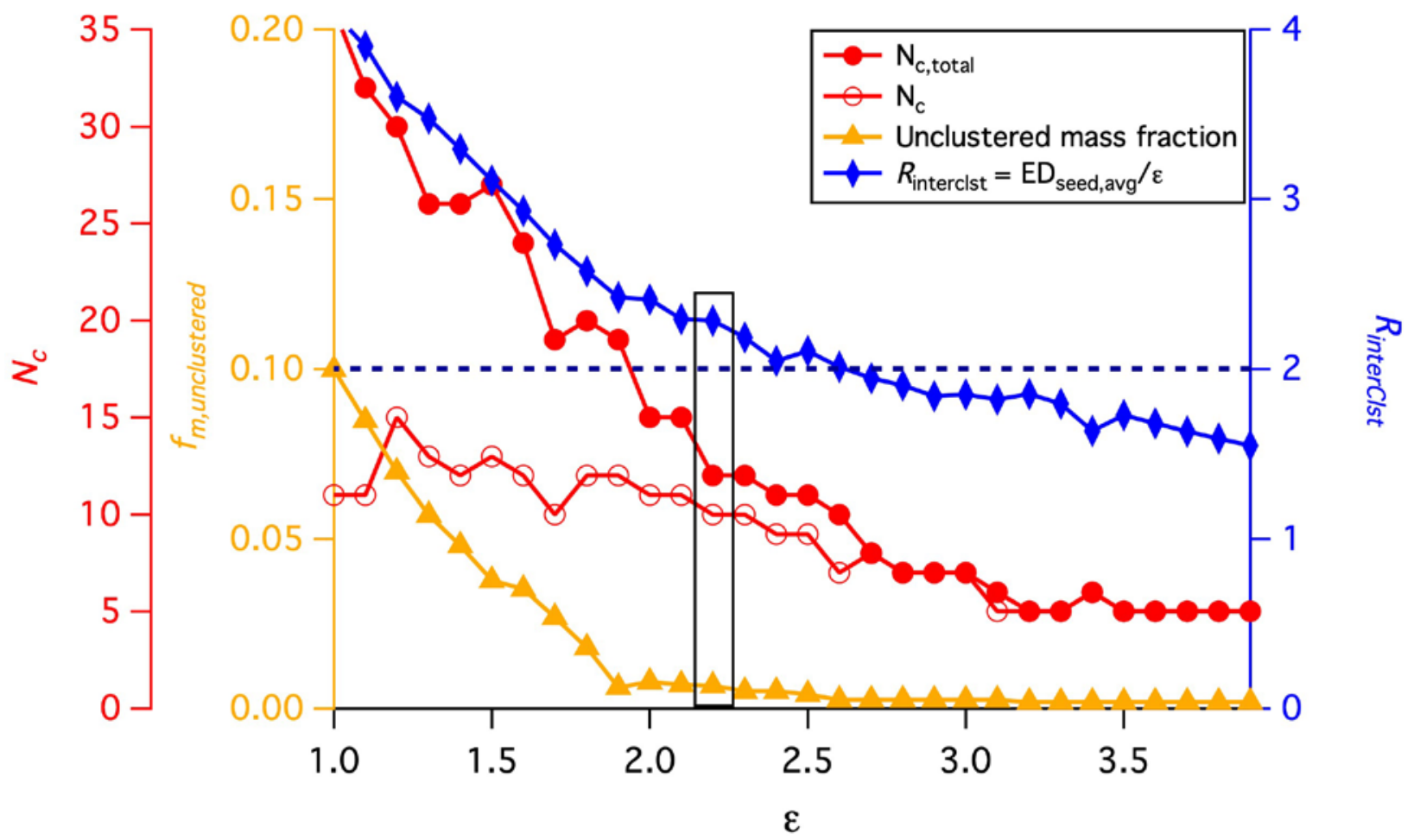

Figure S5. Guidance for determining the optimal $\varepsilon$ for the $\alpha$-pinene $+\mathrm{O}_{3}$ SOA system with no isothermal evaporation for the single clustering approach. The variation of four parameters, $N_{c}, N_{c, t o t a l}, f_{m \text {, unclustered and }}$ $\mathrm{R}_{\text {interclst }}$ are shown as a function of the distance criterion $\varepsilon$. The black horizontal dashed line is guide judgement for $\mathrm{R}_{\text {interclst }} \geq 2$. The values highlighted by a rectangle are the values corresponding to the optimal $\varepsilon$ used for the following clustering analysis. 
a)
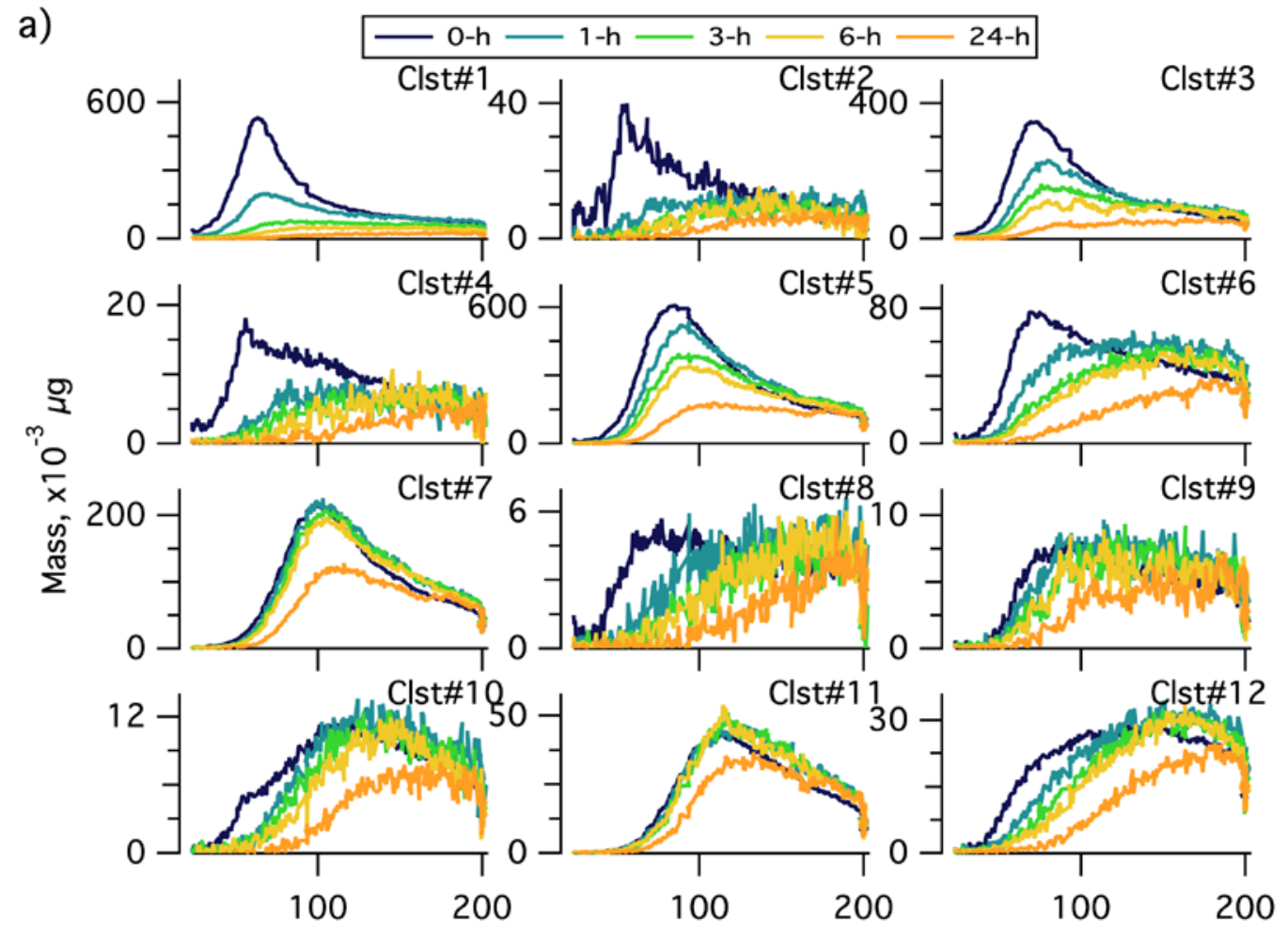

b)

Desorption temperature, ${ }^{\circ} \mathrm{C}$
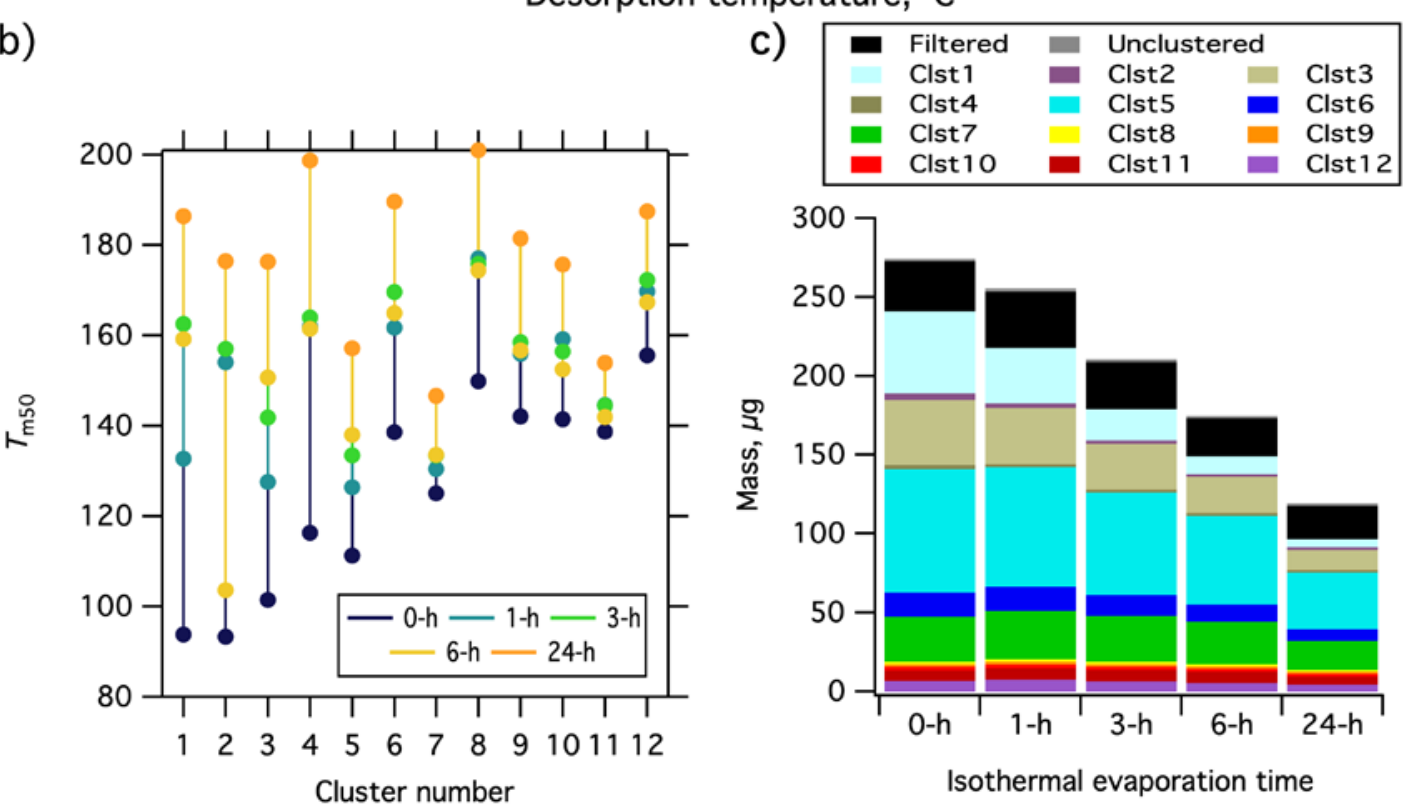

Figure S6 Similar to Figure 12 but presented in the absolute sense. (a) Comparison of the summed thermograms of the 12 clusters of 0 -h wait (navy), 1-h wait (blue), 3-h wait (green), 6-h wait (yellow) and 24-h wait (orange) experiments. (b) Changes in the $T_{m 50}$ for all the clusters calculated from the summed thermograms, with the same color scheme as (a). (c) Absolute mass of each cluster for each experiment, including the summed mass of filtered out ions (black) and unclustered ions (gray). 
Table S5 Chemical characteristics of each cluster identified in the $\alpha$-pinene $+\mathrm{O}_{3}+$ Evaporation SOA system from the single-clustering approach, using the no-wait experiment as the reference.

Table S5-1.

\begin{tabular}{|c|c|c|c|c|c|c|c|c|c|c|}
\hline $\begin{array}{l}\text { Cluster } \\
\#\end{array}$ & $\begin{array}{c}\text { Expt. \#4 } \\
\text { ( } \alpha \text {-pinene+ } \mathrm{O}_{3}+\text { Evap.) } \\
\text { Molecular Formula }\end{array}$ & $O: C$ & $\mathrm{H}: \mathrm{C}$ & MW & $\mathrm{Oh}$ & $1 \mathrm{~h}$ & $\begin{array}{l}3 \mathrm{~h} \\
\text { Mass \% }\end{array}$ & $6 \mathrm{~h}$ & $24 \mathrm{~h}$ & \# lons \\
\hline 1 & $\mathrm{C}_{9.8} \mathrm{H}_{14.0} \mathrm{O}_{5.7} \mathrm{~N}_{0.0}$ & 0.58 & 1.42 & 222.8 & 19.0 & 13.7 & 9.5 & 7.0 & 4.8 & 2 \\
\hline 2 & $\mathrm{C}_{9.0} \mathrm{H}_{16.0} \mathrm{O}_{3.0} \mathrm{~N}_{0.0}$ & 0.33 & 1.78 & 172.0 & 1.5 & 1.1 & 0.9 & 0.3 & 1.0 & 1 \\
\hline 3 & $\mathrm{C}_{8.7} \mathrm{H}_{14.1} \mathrm{O}_{4.8} \mathrm{~N}_{0.0}$ & 0.54 & 1.61 & 195.3 & 15.0 & 14.0 & 14.0 & 13.5 & 11.3 & 9 \\
\hline 4 & $\mathrm{C}_{6.5} \mathrm{H}_{11.1} \mathrm{O}_{4.0} \mathrm{~N}_{0.0}$ & 0.61 & 1.69 & 153.1 & 1.0 & 0.7 & 0.7 & 0.7 & 0.8 & 2 \\
\hline 5 & $\mathrm{C}_{8.9} \mathrm{H}_{14.0} \mathrm{O}_{6.1} \mathrm{~N}_{0.0}$ & 0.69 & 1.58 & 218.4 & 28.5 & 29.6 & 31.0 & 32.1 & 30.3 & 35 \\
\hline 6 & $\mathrm{C}_{8.7} \mathrm{H}_{12.2} \mathrm{O}_{4.9} \mathrm{~N}_{0.0}$ & 0.56 & 1.40 & 195.0 & 5.6 & 6.1 & 6.2 & 6.3 & 6.2 & 8 \\
\hline 7 & $\mathrm{C}_{12.6} \mathrm{H}_{23.5} \mathrm{O}_{7.8} \mathrm{~N}_{0.0}$ & 0.62 & 1.86 & 299.5 & 10.4 & 12.1 & 14.1 & 15.8 & 16.4 & 34 \\
\hline 8 & $\mathrm{C}_{6.0} \mathrm{H}_{8.0} \mathrm{O}_{4.0} \mathrm{~N}_{0.0}$ & 0.67 & 1.33 & 144.0 & 0.46 & 0.5 & 0.4 & 0.5 & 0.6 & 1 \\
\hline 9 & $\mathrm{C}_{6.7} \mathrm{H}_{13.9} \mathrm{O}_{8.2} \mathrm{~N}_{0.0}$ & 1.22 & 2.07 & 225.5 & 0.6 & 0.7 & 0.7 & 0.8 & 0.9 & 3 \\
\hline 10 & $\mathrm{C}_{5.1} \mathrm{H}_{8.1} \mathrm{O}_{3.0} \mathrm{~N}_{0.0}$ & 0.59 & 1.60 & 117.3 & 0.8 & 1.0 & 1.0 & 0.9 & 1.0 & 4 \\
\hline 11 & $\mathrm{C}_{13.7} \mathrm{H}_{24.7} \mathrm{O}_{8.5} \mathrm{~N}_{0.0}$ & 0.62 & 1.80 & 325.1 & 2.4 & 2.8 & 3.4 & 4.0 & 4.6 & 8 \\
\hline 12 & $\mathrm{C}_{81} \mathrm{H}_{10.8} \mathrm{O}_{41} \mathrm{~N}_{00}$ & 0.50 & 1.34 & 173.6 & 2.5 & 2.9 & 3.1 & 3.2 & 3.6 & 5 \\
\hline \multicolumn{5}{|c|}{ Unclustered } & 0.7 & 0.8 & 0.8 & 0.8 & 0.8 & 5 \\
\hline \multicolumn{5}{|l|}{ Filtered } & 11.5 & 14.0 & 14.0 & 13.8 & 17.6 & 185 \\
\hline
\end{tabular}

Table S5-2

\begin{tabular}{|c|c|c|c|c|c|c|c|c|c|c|c|}
\hline Cluster \# & $\begin{array}{c}\text { Expt. \#4 } \\
\text { ( } \alpha \text {-pinene }+\mathrm{O}_{3}+\text { Evap.) } \\
\text { Molecular Formula }\end{array}$ & $\mathrm{Oh}$ & $1 \mathrm{~h}$ & $\begin{array}{l}3 \mathrm{~h} \\
T_{\mathrm{m}, 50} \\
\end{array}$ & $6 \mathrm{~h}$ & $24 \mathrm{~h}$ & $\mathrm{Oh}$ & $1 \mathrm{~h}$ & $3 \mathrm{~h}$ & $6 \mathrm{~h}$ & $24 \mathrm{~h}$ \\
\hline 1 & $\mathrm{C}_{9.8} \mathrm{H}_{14.0} \mathrm{O}_{5.7} \mathrm{~N}_{0.0}$ & 79.6 & 104.3 & 124.4 & 130.6 & 146.4 & 40.1 & 45.9 & 38.3 & 33.4 & 30.1 \\
\hline 2 & $\mathrm{C}_{9.0} \mathrm{H}_{16.0} \mathrm{O}_{3.0} \mathrm{~N}_{0.0}$ & 87.5 & 128.1 & 134.3 & 130.2 & 151.7 & 43.2 & 35.6 & 30.3 & 23.8 & 24.6 \\
\hline 3 & $\mathrm{C}_{8.7} \mathrm{H}_{14.1} \mathrm{O}_{4.8} \mathrm{~N}_{0.0}$ & 93.9 & 107.7 & 117.8 & 126.9 & 141.7 & 33.7 & 40.6 & 38.6 & 34.6 & 32.0 \\
\hline 4 & $\mathrm{C}_{6.5} \mathrm{H}_{11.1} \mathrm{O}_{4.0} \mathrm{~N}_{0.0}$ & 99.6 & 128.1 & 136.5 & 145.6 & 161.7 & 40.9 & 36.7 & 31.6 & 25.4 & 21.9 \\
\hline 5 & $\mathrm{C}_{8.9} \mathrm{H}_{14.0} \mathrm{O}_{6.1} \mathrm{~N}_{0.0}$ & 101.0 & 111.5 & 118.3 & 120.6 & 135.0 & 32.5 & 34.5 & 35.8 & 35.4 & 32.6 \\
\hline 6 & $\mathrm{C}_{8.7} \mathrm{H}_{12.2} \mathrm{O}_{4.9} \mathrm{~N}_{0.0}$ & 111.6 & 131.7 & 140.8 & 141.8 & 156.0 & 42.2 & 33.4 & 29.2 & 28.7 & 25.3 \\
\hline 7 & $\mathrm{C}_{12.6} \mathrm{H}_{23.5} \mathrm{O}_{7.8} \mathrm{~N}_{0.0}$ & 114.6 & 120.0 & 122.1 & 123.3 & 131.6 & 32.0 & 32.0 & 31.7 & 32.4 & 30.5 \\
\hline 8 & $\mathrm{C}_{6.0} \mathrm{H}_{8.0} \mathrm{O}_{4.0} \mathrm{~N}_{0.0}$ & 116.7 & 140.5 & 148.3 & 153.0 & 166.4 & 40.9 & 32.6 & 27.4 & 23.3 & 19.5 \\
\hline 9 & $\mathrm{C}_{6.7} \mathrm{H}_{13.9} \mathrm{O}_{8.2} \mathrm{~N}_{0.0}$ & 118.6 & 129.0 & 135.7 & 132.6 & 148.5 & 37.6 & 34.3 & 28.8 & 32.0 & 30.4 \\
\hline 10 & $\mathrm{C}_{5.1} \mathrm{H}_{8.1} \mathrm{O}_{3.0} \mathrm{~N}_{0.0}$ & 126.8 & 138.5 & 138.0 & 141.4 & 155.5 & 31.4 & 28.0 & 28.3 & 23.3 & 21.1 \\
\hline 11 & $\mathrm{C}_{13.7} \mathrm{H}_{24.7} \mathrm{O}_{8.5} \mathrm{~N}_{0.0}$ & 127.4 & 131.6 & 133.0 & 131.2 & 140.2 & 29.0 & 29.0 & 28.0 & 27.8 & 26.4 \\
\hline 12 & $\mathrm{C}_{8.1} \mathrm{H}_{10.8} \mathrm{O}_{4.1} \mathrm{~N}_{0.0}$ & 130.5 & 141.9 & 146.4 & 148.3 & 158.4 & 33.0 & 29.7 & 27.4 & 25.1 & 23.2 \\
\hline $\begin{array}{l}\text { Unclustered } \\
\text { Filtered }\end{array}$ & & & & & & & & & & & \\
\hline
\end{tabular}




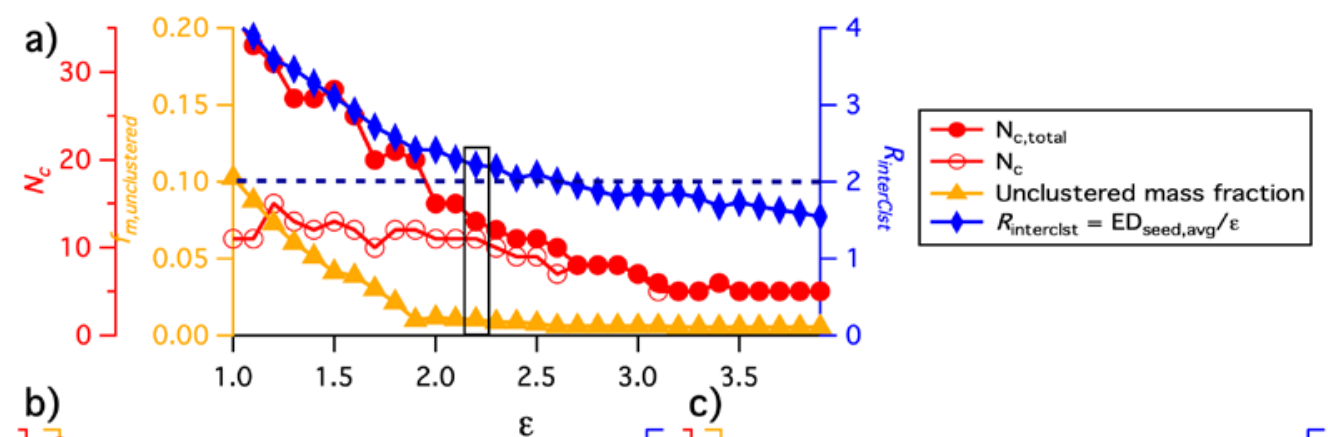

b)

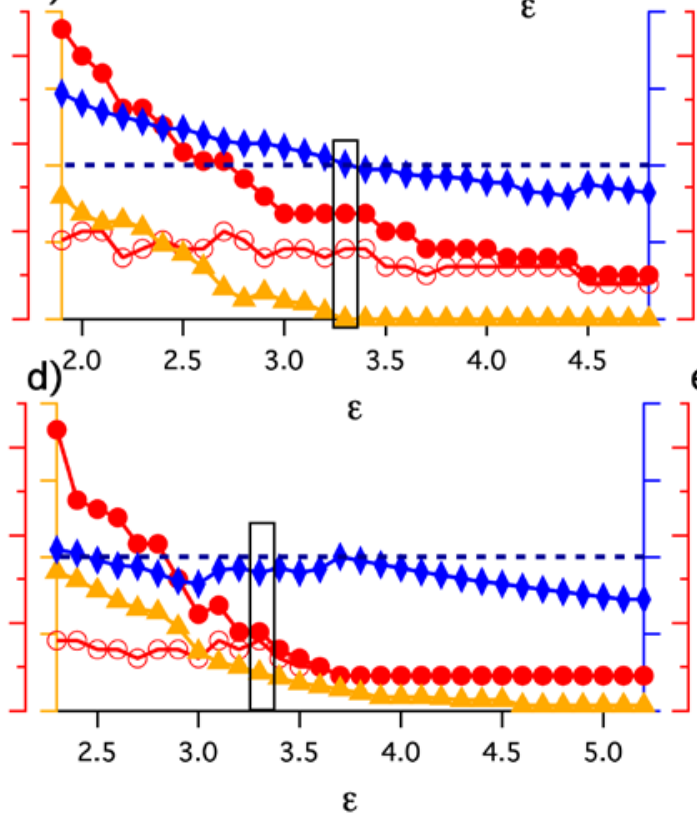

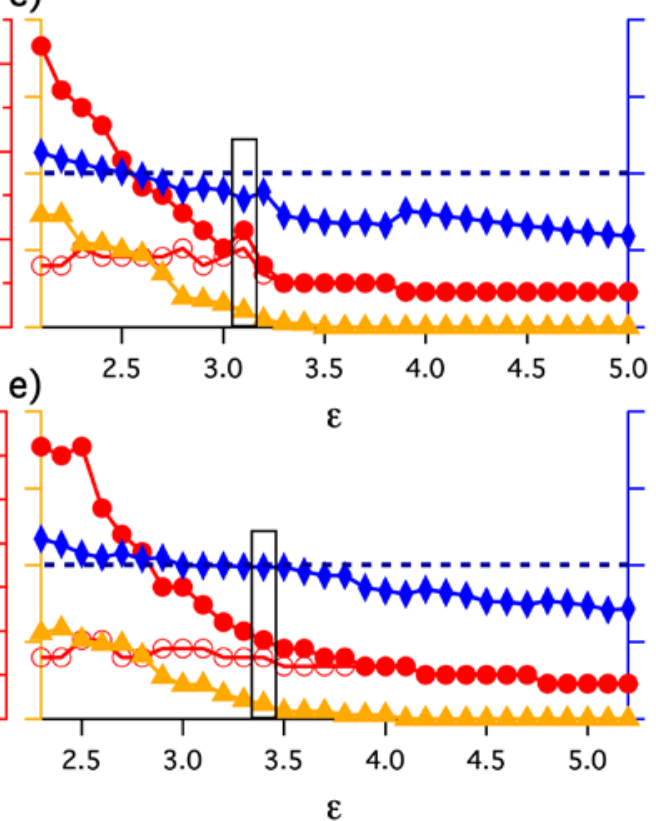

Figure S7. Guidance for determining the optimal $\varepsilon$ for the $\alpha$-pinene $+\mathrm{O}_{3} \mathrm{SOA}$ system with (a) no isothermal evaporation (b) $1 \mathrm{hr}$ (c) $3 \mathrm{hrs}$ (d) $6 \mathrm{hrs}$ and (e) $24 \mathrm{hrs}$ of isothermal evaporation for the multiple clustering approach. The variation of four parameters, $N_{c}, N_{c, t o t a l}, f_{m \text {, unclustered }}$ and $R_{\text {interclst }}$ are shown as a function of the distance criterion $\varepsilon$. The black horizontal dashed line is guide judgement for $R_{\text {interclst }} \geq 2$. The values highlighted by a rectangle are the values corresponding to the optimal $\varepsilon$ used for the following clustering analysis. 
Table $\mathbf{S 6}$ Chemical characteristics of each cluster identified in the $\alpha$-pinene $+\mathrm{O}_{3}+$ Evaporation SOA system for five different isothermal evaporation conditions. The multiple clustering approach is used so each evaporation experiment is clustered independently.

Table S6-1. Results for the $0 \mathrm{~h}$ isothermal evaporation experiment.

\begin{tabular}{lllllllll}
\hline Cluster \# & $\begin{array}{c}\text { Expt. \#4a } \\
\text { ( } \alpha \text {-pinene+ } \mathrm{O}_{3}+\text { O-h Evap.) } \\
\text { Molecular Formula }\end{array}$ & $\mathrm{O}: \mathrm{C}$ & $\mathrm{H}: \mathrm{C}$ & Mass \% & \# lons & $T_{\mathrm{m}, 50}$ & $T_{\mathrm{m}, 75}$ & $\Delta T$ \\
\hline 1 & $\mathrm{C}_{9.8} \mathrm{H}_{14.0} \mathrm{O}_{5.7} \mathrm{~N}_{0.0}$ & 0.58 & 1.42 & 18.9 & 2 & 79.6 & 119.7 & 40.1 \\
2 & $\mathrm{C}_{9.0} \mathrm{H}_{16.0} \mathrm{O}_{3.0} \mathrm{~N}_{0.0}$ & 0.33 & 1.78 & 1.4 & 1 & 87.5 & 130.8 & 43.3 \\
3 & $\mathrm{C}_{8.5} \mathrm{H}_{13.6} \mathrm{O}_{4.9} \mathrm{~N}_{0.0}$ & 0.58 & 1.61 & 12.2 & 8 & 93.6 & 124.3 & 30.7 \\
4 & $\mathrm{C}_{10.9} \mathrm{H}_{18.5} \mathrm{O}_{3.7} \mathrm{~N}_{0.0}$ & 0.34 & 1.69 & 3.2 & 2 & 97.5 & 140.7 & 43.2 \\
5 & $\mathrm{C}_{6.5} \mathrm{H}_{11.1} \mathrm{O}_{4.0} \mathrm{~N}_{0.0}$ & 0.61 & 1.69 & 1.0 & 2 & 99.6 & 140.5 & 40.9 \\
6 & $\mathrm{C}_{8.9} \mathrm{H}_{14.0} \mathrm{O}_{6.1} \mathrm{~N}_{0.0}$ & 0.69 & 1.58 & 28.2 & 35 & 101.0 & 133.5 & 32.5 \\
7 & $\mathrm{C}_{8.7} \mathrm{H}_{12.2} \mathrm{O}_{4.9} \mathrm{~N}_{0.0}$ & 0.56 & 1.40 & 5.6 & 8 & 111.6 & 153.8 & 42.2 \\
8 & $\mathrm{C}_{12.6} \mathrm{H}_{23.5} \mathrm{O}_{7.8} \mathrm{~N}_{0.0}$ & 0.62 & 1.86 & 10.3 & 34 & 114.6 & 146.5 & 31.9 \\
9 & $\mathrm{C}_{6.0} \mathrm{H}_{8.0} \mathrm{O}_{4.0} \mathrm{~N}_{0.0}$ & 0.67 & 1.33 & 0.5 & 1 & 116.7 & 157.6 & 40.9 \\
10 & $\mathrm{C}_{6.7} \mathrm{H}_{13.9} \mathrm{O}_{8.2} \mathrm{~N}_{0.0}$ & 1.22 & 2.07 & 0.6 & 3 & 118.6 & 156.2 & 37.6 \\
11 & $\mathrm{C}_{5.1} \mathrm{H}_{8.1} \mathrm{O}_{3.0} \mathrm{~N}_{0.0}$ & 0.59 & 1.60 & 0.8 & 4 & 126.8 & 158.2 & 31.4 \\
12 & $\mathrm{C}_{13.7} \mathrm{H}_{24.7} \mathrm{O}_{8.5} \mathrm{~N}_{0.0}$ & 0.62 & 1.80 & 2.3 & 8 & 127.4 & 156.3 & 28.9 \\
13 & $\mathrm{C}_{8.1} \mathrm{H}_{10.8} \mathrm{O}_{4.1} \mathrm{~N}_{0.0}$ & 0.50 & 1.34 & 2.5 & 5 & 130.5 & 163.5 & 33.0 \\
Unclustered & & & & 1.0 & 7 & & & \\
Filtered & & & & 11.4 & 191 & & & \\
\hline
\end{tabular}

Table S6-2. Results for the $1 \mathrm{~h}$ isothermal evaporation experiment.

\begin{tabular}{lllllllll}
\hline Cluster \# & $\begin{array}{c}\text { Expt. \#4b } \\
\text { ( } \alpha \text {-pinene+ } \mathrm{O}_{3}+\text { 1-h Evap.) } \\
\text { Molecular Formula }\end{array}$ & $\mathrm{O}: \mathrm{C}$ & $\mathrm{H}: \mathrm{C}$ & Mass \% & \# lons & $T_{\mathrm{m}, 50}$ & $T_{\mathrm{m}, 75}$ & $\Delta T$ \\
\hline 1 & $\mathrm{C}_{2.0} \mathrm{H}_{4.0} \mathrm{O}_{3.0} \mathrm{~N}_{0.0}$ & 1.50 & 2.00 & 1.3 & 1 & 85.4 & 131.6 & 46.2 \\
2 & $\mathrm{C}_{9.4} \mathrm{H}_{16.7} \mathrm{O}_{5.2} \mathrm{~N}_{0.0}$ & 0.55 & 1.79 & 1.6 & 3 & 96.1 & 125.8 & 29.7 \\
3 & $\mathrm{C}_{9.1} \mathrm{H}_{13.5} \mathrm{O}_{5.5} \mathrm{~N}_{0.0}$ & 0.61 & 1.48 & 20.1 & 6 & 103.3 & 146.5 & 43.2 \\
4 & $\mathrm{C}_{9.6} \mathrm{H}_{15.7} \mathrm{O}_{6.2} \mathrm{~N}_{0.0}$ & 0.65 & 1.63 & 36.5 & 48 & 113.9 & 148.4 & 34.5 \\
5 & $\mathrm{C}_{16.0} \mathrm{H}_{32.0} \mathrm{O}_{2.0} \mathrm{~N}_{0.0}$ & 0.12 & 2.00 & 0.9 & 1 & 118.3 & 181.6 & 63.3 \\
6 & $\mathrm{C}_{9.0} \mathrm{H}_{14.0} \mathrm{O}_{4.0} \mathrm{~N}_{0.0}$ & 0.444 & 1.56 & 2.6 & 1 & 120.0 & 162.0 & 42.0 \\
7 & $\mathrm{C}_{14.5} \mathrm{H}_{28.1} \mathrm{O}_{8.7} \mathrm{~N}_{0.0}$ & 0.60 & 1.94 & 4.3 & 10 & 127.1 & 155.5 & 28.4 \\
8 & $\mathrm{C}_{8.5} \mathrm{H}_{12.5} \mathrm{O}_{5.4} \mathrm{~N}_{0.0}$ & 0.64 & 1.47 & 4.3 & 6 & 130.8 & 164.3 & 33.5 \\
9 & $\mathrm{C}_{11.1} \mathrm{H}_{20.9} \mathrm{O}_{8.9} \mathrm{~N}_{0.0}$ & 0.80 & 1.88 & 1.4 & 5 & 132.3 & 161.8 & 29.5 \\
10 & $\mathrm{C}_{7.4} \mathrm{H}_{10.1} \mathrm{O}_{3.9} \mathrm{~N}_{0.0}$ & 0.52 & 1.36 & 8.6 & 18 & 140.9 & 171.0 & 30.1 \\
11 & $\mathrm{C}_{7.0} \mathrm{H}_{8.0} \mathrm{O}_{4.0} \mathrm{~N}_{0.0}$ & 0.57 & 1.14 & 0.6 & 1 & 156.8 & 181.4 & 24.6 \\
12 & $\mathrm{C}_{17.3} \mathrm{H}_{34.0} \mathrm{O}_{2.1} \mathrm{~N}_{0.0}$ & 0.12 & 1.97 & 1.1 & 2 & 189.2 & 199.1 & 9.0 \\
Unclustered & & & & 0.0 & 0 & & & \\
Filtered & & & & 16.5 & 210 & & & \\
\hline
\end{tabular}


Table S6-3. Results for the $3 \mathrm{~h}$ isothermal evaporation experiment.

\begin{tabular}{lllllllll}
\hline Cluster \# & $\begin{array}{c}\text { Expt. \#4c } \\
\text { (a-pinene+ } \mathrm{O}_{3}+\text { 3-h Evap.) } \\
\text { Molecular Formula }\end{array}$ & $\mathrm{O}: \mathrm{C}$ & $\mathrm{H}: \mathrm{C}$ & Mass \% & \# lons & $T_{\mathrm{m}, 50}$ & $T_{\mathrm{m}, 75}$ & $\Delta T$ \\
\hline 1 & $\mathrm{C}_{10.0} \mathrm{H}_{18.0} \mathrm{O}_{5.0} \mathrm{~N}_{0.0}$ & 0.50 & 1.80 & 0.5 & 1 & 97.9 & 133.4 & 35.5 \\
2 & $\mathrm{C}_{9.4} \mathrm{H}_{14.9} \mathrm{O}_{6.0} \mathrm{~N}_{0.0}$ & 0.63 & 1.58 & 16.7 & 13 & 111.8 & 146.2 & 34.4 \\
3 & $\mathrm{C}_{13.1} \mathrm{H}_{23.8} \mathrm{O}_{7.5} \mathrm{~N}_{0.0}$ & 0.57 & 1.81 & 17.7 & 31 & 121.4 & 153.3 & 31.9 \\
4 & $\mathrm{C}_{8.6} \mathrm{H}_{12.8} \mathrm{O}_{5.3} \mathrm{~N}_{0.0}$ & 0.62 & 1.49 & 23.1 & 9 & 121.6 & 159.0 & 37.4 \\
5 & $\mathrm{C}_{15.5} \mathrm{H}_{26.8} \mathrm{O}_{5.9} \mathrm{~N}_{0.0}$ & 0.38 & 1.73 & 0.4 & 2 & 123.1 & 156.6 & 33.5 \\
6 & $\mathrm{C}_{8.4} \mathrm{H}_{12.9} \mathrm{O}_{6.9} \mathrm{~N}_{0.0}$ & 0.81 & 1.53 & 4.8 & 8 & 128.3 & 159.0 & 30.7 \\
7 & $\mathrm{C}_{11.1} \mathrm{H}_{21.9} \mathrm{O}_{8.8} \mathrm{~N}_{0.0}$ & 0.79 & 1.97 & 1.5 & 5 & 133.4 & 162.1 & 28.7 \\
8 & $\mathrm{C}_{9.0} \mathrm{H}_{16.0} \mathrm{O}_{3.0} \mathrm{~N}_{0.0}$ & 0.33 & 1.78 & 0.9 & 1 & 134.3 & 164.7 & 30.4 \\
9 & $\mathrm{C}_{7.1} \mathrm{H}_{12.8} \mathrm{O}_{5.7} \mathrm{~N}_{0.0}$ & 0.81 & 1.80 & 4.3 & 3 & 134.9 & 169.3 & 34.4 \\
10 & $\mathrm{C}_{7.9} \mathrm{H}_{10.8} \mathrm{O}_{4.2} \mathrm{~N}_{0.0}$ & 0.53 & 1.36 & 13.0 & 22 & 143.4 & 171.6 & 28.2 \\
11 & $\mathrm{C}_{8.3 .} \mathrm{H}_{10.6} \mathrm{O}_{4.0} \mathrm{~N}_{0.0}$ & 0.48 & 1.28 & 1.7 & 2 & 156.1 & 181.3 & 25.2 \\
Unclustered & & & & 1.1 & 5 & & & \\
Filtered & & & & 14.3 & 205 & & & \\
\hline
\end{tabular}

Table S6-4. Results for the $6 \mathrm{~h}$ isothermal evaporation experiment.

\begin{tabular}{|c|c|c|c|c|c|c|c|c|}
\hline Cluster \# & $\begin{array}{c}\text { Expt. \#4d } \\
\text { ( } \alpha \text {-pinene }+\mathrm{O}_{3}+6 \text {-h Evap.) } \\
\text { Molecular Formula }\end{array}$ & $\mathrm{O}: \mathrm{C}$ & $\mathrm{H}: \mathrm{C}$ & Mass \% & \# lons & $T_{\mathrm{m}, 50}$ & $T_{\mathrm{m}, 75}$ & $\Delta T$ \\
\hline 1 & $\mathrm{C}_{9.8} \mathrm{H}_{14.1} \mathrm{O}_{6.9} \mathrm{~N}_{0.0}$ & 0.70 & 1.44 & 4.9 & 3 & 108.2 & 138.3 & 30.1 \\
\hline 2 & $\mathrm{C}_{9.3} \mathrm{H}_{14.8} \mathrm{O}_{5.1} \mathrm{~N}_{0.0}$ & 0.55 & 1.59 & 4.5 & 2 & 119.7 & 157.1 & 37.4 \\
\hline 3 & $\mathrm{C}_{11.9} \mathrm{H}_{21.0} \mathrm{O}_{7.3} \mathrm{~N}_{0.0}$ & 0.61 & 1.76 & 29.2 & 36 & 121.9 & 154.2 & 32.3 \\
\hline 4 & $\mathrm{C}_{8.5} \mathrm{H}_{13.1} \mathrm{O}_{5.4} \mathrm{~N}_{0.0}$ & 0.63 & 1.54 & 24.0 & 13 & 128.8 & 163.3 & 34.5 \\
\hline 5 & $\mathrm{C}_{11.5} \mathrm{H}_{23.0} \mathrm{O}_{9.5} \mathrm{~N}_{0.0}$ & 0.83 & 2.00 & 0.6 & 2 & 129.2 & 161.7 & 32.5 \\
\hline 6 & $\mathrm{C}_{8.1} \mathrm{H}_{12.7} \mathrm{O}_{6.6} \mathrm{~N}_{0.0}$ & 0.82 & 1.58 & 5.5 & 11 & 132.9 & 164.9 & 32.0 \\
\hline 7 & $\mathrm{C}_{11.0} \mathrm{H}_{16.0} \mathrm{O}_{5.0} \mathrm{~N}_{0.0}$ & 0.45 & 1.45 & 0.4 & 1 & 144.4 & 172.3 & 27.9 \\
\hline 8 & $\mathrm{C}_{7.7} \mathrm{H}_{10.6} \mathrm{O}_{4.0} \mathrm{~N}_{0.0}$ & 0.52 & 1.39 & 15.0 & 25 & 147.2 & 173.0 & 25.8 \\
\hline 9 & $\mathrm{C}_{2.7} \mathrm{H}_{5,3} \mathrm{O}_{3,3} \mathrm{~N}_{0.0}$ & 1.25 & 2.00 & 0.6 & 2 & 183.9 & 194.9 & 11.0 \\
\hline Unclustered & & & & 2.5 & 11 & & & \\
\hline Filtered & & & & 12.8 & 203 & & & \\
\hline
\end{tabular}

Table S6-5. Results for the $24 \mathrm{~h}$ isothermal evaporation experiment.

\begin{tabular}{|c|c|c|c|c|c|c|c|c|}
\hline Cluster \# & $\begin{array}{c}\text { Expt. \#4e } \\
\text { ( } \alpha \text {-pinene }+\mathrm{O}_{3}+24-\mathrm{h} \text { Evap.) } \\
\text { Molecular Formula }\end{array}$ & $\mathrm{O}: \mathrm{C}$ & $\mathrm{H}: \mathrm{C}$ & Mass \% & \# lons & $T_{m, 50}$ & $T_{\mathrm{m}, 75}$ & $\Delta T$ \\
\hline 1 & $\mathrm{C}_{12.1} \mathrm{H}_{21.2} \mathrm{O}_{7.2} \mathrm{~N}_{0.0}$ & 0.60 & 1.75 & 25.4 & 26 & 130.0 & 161.1 & 31.1 \\
\hline 2 & $\mathrm{C}_{7.0} \mathrm{H}_{16.0} \mathrm{O}_{9.0} \mathrm{~N}_{0.0}$ & 1.29 & 2.29 & 0.6 & 1 & 133.9 & 166.7 & 32.8 \\
\hline 3 & $\mathrm{C}_{11.3} \mathrm{H}_{17.9} \mathrm{O}_{6.7} \mathrm{~N}_{0.0}$ & 0.59 & 1.58 & 1.7 & 4 & 137.7 & 167.7 & 30.0 \\
\hline 4 & $\mathrm{C}_{11.9} \mathrm{H}_{20.0} \mathrm{O}_{8.2} \mathrm{~N}_{0.0}$ & 0.69 & 1.68 & 4.4 & 10 & 140.1 & 167.6 & 27.5 \\
\hline 5 & $\mathrm{C}_{8.9} \mathrm{H}_{13.8} \mathrm{O}_{5.5} \mathrm{~N}_{0.0}$ & 0.62 & 1.54 & 16.1 & 8 & 140.4 & 171.5 & 31.1 \\
\hline 6 & $\mathrm{C}_{7.9} \mathrm{H}_{12.0} \mathrm{O}_{4.6} \mathrm{~N}_{0.0}$ & 0.58 & 1.51 & 20.3 & 23 & 154.5 & 179.0 & 24.5 \\
\hline 7 & $\mathrm{C}_{5.2} \mathrm{H}_{7.1} \mathrm{O}_{3.4} \mathrm{~N}_{0.0}$ & 0.66 & 1.37 & 12.2 & 17 & 168.0 & 186.3 & 18.3 \\
\hline 8 & $\mathrm{C}_{16.0} \mathrm{H}_{32.0} \mathrm{O}_{2.0} \mathrm{~N}_{0.0}$ & 0.12 & 2.00 & 1.0 & 1 & 177.9 & 197.9 & 20.0 \\
\hline 9 & $\mathrm{C}_{17.5} \mathrm{H}_{35.0} \mathrm{O}_{2.1} \mathrm{~N}_{0.0}$ & 0.12 & 2.00 & 1.2 & 2 & 198.7 & 199.3 & 0.6 \\
\hline Unclustered & & & & 1.0 & 4 & & & \\
\hline Filtered & & & & 16.1 & 213 & & & \\
\hline
\end{tabular}




\section{Supplemental References}

D'Ambro, E. L., Schobesberger, S., Zaveri, R. A., Shilling, J. E., Lee, B. H., Lopez-Hilfiker, F. D., Mohr, C., and Thornton, J. A.: Isothermal Evaporation of alpha-Pinene Ozonolysis SOA: Volatility, Phase State, and Oligomeric Composition, Acs Earth Space Chem, 2, 1058-1067, https://doi.org/10.1021/acsearthspacechem.8b00084, 2018.

Isaacman-VanWertz, G., Massoli, P., O'Brien, R. E., Nowak, J. B., Canagaratna, M. R., Jayne, J. T., Worsnop, D. R., Su, L., Knopf, D. A., Misztal, P. K., Arata, C., Goldstein, A. H., and Kroll, J. H.: Using advanced mass spectrometry techniques to fully characterize atmospheric organic carbon: current capabilities and remaining gaps, Faraday Discussions, 200, 579-598, https://doi.org/10.1039/c7fd00021a, 2017.

Lee, B., Lopez-Hilfiker, F. D., D'Ambro, E. L., Zhou, P. T., Boy, M., Petaja, T., Hao, L. Q., Virtanen, A., and Thornton, J. A.: Semi-volatile and highly oxygenated gaseous and particulate organic compounds observed above a boreal forest canopy, Atmospheric Chemistry and Physics, 18, 11547-11562, https://doi.org/10.5194/acp-18-11547-2018, 2018.

Lee, B. H., Lopez-Hilfiker, F. D., Mohr, C., Kurten, T., Worsnop, D. R., and Thornton, J. A.: An lodideAdduct High-Resolution Time-of-Flight Chemical-Ionization Mass Spectrometer: Application to Atmospheric Inorganic and Organic Compounds, Environ Sci Technol, 48, 6309-6317, https://doi.org/10.1021/es500362a, 2014.

Liu, J. M., D'Ambro, E. L., Lee, B. H., Lopez-Hilfiker, F. D., Zaveri, R. A., Rivera-Rios, J. C., Keutsch, F. N., Iyer, S., Kurten, T., Zhang, Z. F., Gold, A., Surratt, J. D., Shilling, J. E., and Thornton, J. A.: Efficient Isoprene Secondary Organic Aerosol Formation from a Non-IEPDX Pathway, Environ Sci Technol, 50, 9872-9880, https://doi.org/10.1021/acs.est.6b01872, 2016.

Liu, S., Shilling, J. E., Song, C., Hiranuma, N., Zaveri, R. A., and Russell, L. M.: Hydrolysis of Organonitrate Functional Groups in Aerosol Particles, Aerosol Science and Technology, 46, 13591369, https://doi.org/10.1080/02786826.2012.716175, 2012.

Lopez-Hilfiker, F. D., Mohr, C., Ehn, M., Rubach, F., Kleist, E., Wildt, J., Mentel, T. F., Lutz, A., Hallquist, M., Worsnop, D., and Thornton, J. A.: A novel method for online analysis of gas and particle composition: description and evaluation of a Filter Inlet for Gases and AEROsols (FIGAERO), Atmospheric Measurement Techniques, 7, 983-1001, https://doi.org/10.5194/amt7-983-2014, 2014. 